\title{
Delivery of multipurpose prevention drug combinations from electrospun nanofibers using composite microarchitectures
}

This article was published in the following Dove Press journal:

International Journal of Nanomedicine

17 June 2014

Number of times this article has been viewed

\section{Anna K Blakney \\ Emily A Krogstad \\ Yonghou $\mathrm{H}$ Jiang \\ Kim A Woodrow}

Department of Bioengineering, University of Washington, Seattle, Washington, USA
Correspondence: Kim A Woodrow William H Foege Building N4IOD, 3720 I5th Ave NE, Seattle,WA 98195-506।, USA

Tel + I 206685683 |

Fax +I 2066853300

Email woodrow@uw.edu
Background: Electrospun drug-eluting fabrics have enormous potential for the delivery of physicochemically diverse drugs in combination by controlling the underlying material chemistry and fabric microarchitecture. However, the rationale for formulating drugs at high drug loading in the same or separate fibers is unknown but has important implications for product development and clinical applications.

Methods: Using a production-scale free-surface electrospinning instrument, we produced electrospun nanofibers with different microscale geometries for the co-delivery of tenofovir (TFV) and levonorgestrel (LNG) - two lead drug candidates for multipurpose prevention of HIV acquisition and unintended pregnancy. We investigated the in vitro drug release of TFV and LNG combinations from composites that deliver the two drugs from the same fiber (combined fibers) or from separate fibers in a stacked or interwoven architecture. For stacked composites, we also examined the role that fabric thickness has on drug-release kinetics. We also measured the cytotoxicity and antiviral activity of the drugs delivered alone and in combination.

Results: Herein, we report on the solution and processing parameters for the free-surface electrospinning of medical fabrics with controlled microarchitecture and high drug loading (up to $20 \mathrm{wt} \%$ ). We observed that in vitro release of the highly water-soluble TFV, but not the water-insoluble LNG, was affected by composite microarchitecture, fabric thickness, and drug content. Finally, we showed that the drug-loaded nanofibers are noncytotoxic and that the antiviral activity of TFV is preserved through the electrospinning process and when combined with LNG.

Conclusion: Electrospun fabrics with high drug loading create multicomponent systems that benefit from the independent control of the nanofibrous microarchitecture. Our findings are significant because they will inform the design and production of composite electrospun fabrics for the co-delivery of physicochemically diverse drugs that may be useful for multipurpose prevention.

Keywords: co-delivery, electrospinning, antiretroviral, contraceptive, microbicide, multipurpose prevention technology

\section{Introduction}

The clinical use of combination drug therapy for treating cancer, ${ }^{1} \mathrm{HIV} / \mathrm{AIDS},{ }^{2}$ and multidrug-resistant bacterial infections ${ }^{3}$ underscores the importance of drug combinations for realizing synergies that enhance treatment efficacy while reducing toxicity and addressing the emergence of drug resistance. ${ }^{4}$ However, the co-delivery of drugs requires strategies for combining physicochemically diverse agents and delivering these combinations to target tissues and cells at therapeutically relevant 
concentrations. ${ }^{5,6}$ To realize the full potential of drug combinations, advances in drug delivery systems must address these challenges.

Electrospun fabrics are an ideal topical delivery system for the co-delivery of multiple agents because of their proven capacity to encapsulate and deliver physicochemically diverse drugs and ability to modulate drug release kinetics over both short and long timeframes. ${ }^{7-9}$ A specific application of electrospun fabrics for topical delivery that demands these versatile product attributes is in the development of multipurpose prevention technologies (MPTs). MPTs are combination drug-delivery systems intended for simultaneous prevention of HIV, other sexually transmitted infections, and unintended pregnancy. ${ }^{10}$ Two leading drug candidates for MPTs that target the prevention of unintended pregnancy and HIV acquisition are tenofovir (TFV), a hydrophilic nucleotide reverse transcriptase inhibitor (NRTI), ${ }^{11}$ and levonorgestrel (LNG), a hydrophobic hormonal contraceptive. ${ }^{12}$ TFV and LNG have physicochemical properties that prevent their easy combination at the high drug loadings that may be required for sustained prevention.

Huang et $\mathrm{al}^{13}$ and Ball et $\mathrm{al}^{14}$ have used electrospun fabrics with variety of antiretroviral drugs but never in combination. Combination drug delivery from electrospun fabrics can be achieved by the co-delivery of multiple drugs encapsulated within the same fiber or into separate fibers. Okuda et al used separate fibers that were combined in a multilayered fiber mat for the sustained delivery of two model drugs. ${ }^{15}$ In contrast, Xu et al encapsulated paclitaxel and doxorubicin hydrochloride in the same polyethylene glycol-polylactic acid fibers and observed asynchronous drug release. ${ }^{16}$ However, in all of these studies to date, less than $1 \mathrm{wt} \%$ of the drug has been used alone or in combination, which makes these systems limited in scope to applications employing drugs with high potency. Even drugs with nanomolar potency are required at a minimum composition of $\geq 10 \mathrm{wt} \%$ to be realistically useful as a medical fabric. To our knowledge no systematic study has been performed to assess the co-delivery of physicochemically diverse drugs at high drug loading in electrospun fabrics with different underlying microarchitectures. Here, we demonstrate using a production scale instrument the feasibility of assembling electrospun fabrics, with different microscale geometries for the co-delivery of a hydrophilic (TFV) and hydrophobic (LNG) drug. We show that medical fabrics with high drug loading benefit from independent control of the nanofibrous microarchitecture to predict drug release kinetics.

\section{Materials and methods Polymer solution preparation}

Polyvinyl alcohol (PVA; MW 85-124 kDa, 87\%-89\% hydrolysis) and LNG were purchased from Sigma-Aldrich Co ( $\mathrm{St}$ Louis, MO, USA). TFV was a generous gift from CONRAD. Alexa Fluor ${ }^{\circledR} 488$ and 555 hydrazide sodium salts were purchased from Thermo Fisher Scientific (Waltham, MA, USA). For all polymer solutions, PVA was dissolved in deionized water at $10 \% \mathrm{wt} / \mathrm{vol}$. Drugs were mixed with polymer solution at $20 \% \mathrm{wt} \mathrm{drug} / \mathrm{wt}$ polymer (for equal-loading fabrics) or $0.0067 \%$ (LNG) and $15.3 \%$ (TFV) wt drug/wt polymer (for relevant daily-dosage fabrics) and stirred for $\geq 6$ hours prior to electrospinning.

\section{Electrospinning}

Polymer solutions were electrospun on a Nanospider ${ }^{\mathrm{TM}}$ Production Line NS 1WS500U (Elmarco, Inc, Morrisville, $\mathrm{NC}$, USA) free-surface electrospinning instrument using the following processing conditions unless otherwise specified: $160 \mathrm{~mm}$ wire electrode distance, $-25 \mathrm{kV}$ collecting electrode, $60 \mathrm{kV}$ spinning electrode, and $250 \mathrm{~mm}$ cartridge traveling distance. All microarchitectures were prepared using $15 \mathrm{~mL}$ batch volumes in the single $20 \mathrm{~mL}$ cartridge (Figure 1A), unless otherwise noted. For the stacked composite, we electrospun $7.5 \mathrm{~mL}$ of a $\mathrm{LNG}$ polymer solution followed by $7.5 \mathrm{~mL}$ of a TFV polymer solution. Interwoven composites were prepared similarly except the volumes were separated into different reservoirs of the $40 \mathrm{~mL}$ dual cartridge (Figure 1B). For the fluorescent fibers, LNG and TFV were combined with $0.025 \% \mathrm{wt} / \mathrm{vol}$ of Alexa Fluor $^{\circledR} 488$ and 555, respectively, and collected onto a glass slide. To prepare fabrics of different thicknesses, we decreased the travel distance of the cartridge.

\section{Solution, fiber, and fabric characterization}

Density, conductivity, surface tension, viscosity, and $\mathrm{pH}$ were measured in triplicate (unless otherwise stated) for all solutions prior to electrospinning. Density was measured by taking the mass of a known volume of solution. Conductivity and $\mathrm{pH}$ were measured using an Orion Star ${ }^{\mathrm{TM}} \mathrm{A} 212$ conductivity meter and Orion Star A111 pH meter (both Thermo Fisher Scientific). Surface tension was measured using an AquaPi surface tensiometer (Kibron Inc, Helsinki, Finland). Viscosity was measured using a TA Instruments AR-G2 rheometer (New Castle, DE, USA) at a constant strain of $4 \%$ with a cone (angle $=1^{\circ} 58^{\prime} 48^{\prime \prime}$, diameter $=40 \mathrm{~mm}$ ) and plate geometry.

For the scanning electron microscopy (SEM) images, fabrics were sputter coated with a mixture of gold and 
A

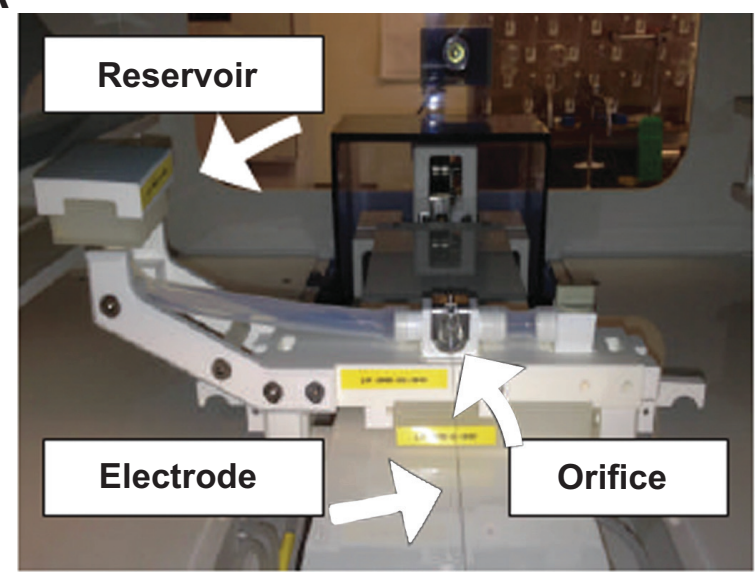

Single $20 \mathrm{~mL}$ cartridge
B

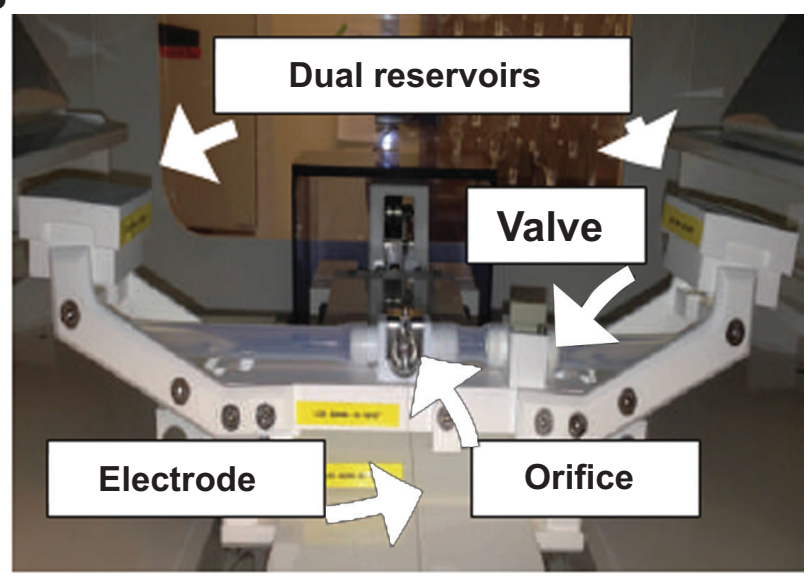

Dual $20 \mathrm{~mL}$ cartridge

Figure I Photographs of the Nanospider TM Production Line NS IWS500U (Elmarco, Inc, Morrisville, NC, USA) cartridges used to fabricate tenofovir (TFV) and levonorgestrel (LNG) medical fabrics. (A) A single $20 \mathrm{~mL}$ reservoir was used to electrospin blank, LNG-only, TFV-only, stacked, and combined fibers. (B) A dual $20 \mathrm{~mL}$ reservoir was used to electrospin interwoven fibers. Images depict the reservoir housing for the electrospinning polymer solution, which drains via the clear tubing toward the metal orifice where the solution is deposited onto the wire electrode.

palladium for 90 seconds and imaged at magnifications of $500 \times$ and 5,000× using a Sirion SEM at the University of Washington (UW) Nanotechnology User Facility. Crosssectional SEM images were acquired by freezing the fabrics in liquid nitrogen for $\sim 30$ seconds and then abruptly fracturing the fabrics to provide a clean break. Image $\mathrm{J}^{17}$ software was used to determine fiber diameter by measuring 45 random fibers.

Thermograms were generated using an Auto Q20 differential scanning calorimeter (TA Instruments at the UW Materials Science Engineering User Facility) for the purpose of calculating the relative crystallinity of drugs in the electrospun fabrics. Samples $(5 \mathrm{mg}$ ) were prepared in an aluminum pan $\left(\mathrm{T}_{\text {zero }}\right)$ and heated at a constant ramp from $10^{\circ} \mathrm{C}$ to $350^{\circ} \mathrm{C}$ with a sampling interval of $1 \mathrm{~s} /$ point. Heat flow was normalized to sample weight. TA Universal Analysis software was used for both rheometry and differential scanning calorimetry data collection and analysis. The mechanical properties of electrospun fabrics were measured using an Instron ${ }^{\circledR} 5543$ Single Column System (Norwood, MA, USA) with a $10 \mathrm{~N}$ load cell, a strain rate of 0.01 /second, and a single-column, screw-driven apparatus at $20^{\circ} \mathrm{C}$ and relative humidity of $40 \%$. The mechanical testing was performed according to method ASTM D5034-95, with a dogbone sample shape (ASTM D1708-96), with length $22 \mathrm{~mm}$, width $5 \mathrm{~mm}$, and variable thickness. The Young's modulus was calculated by analyzing the linear region of the resulting stress versus strain curve, and the tensile strength was calculated by analyzing the peak stress that the sample was able to endure. The encapsulation efficiency (EE), percent yield, productivity, and relative crystallinity ${ }^{18}$ were calculated using the following equations:

$$
\mathrm{EE}=100 \times \frac{\text { Actual mass drug in fibers }}{\text { Expected mass drug in fibers }}
$$

$$
\% \text { yield }=100 \times \frac{\text { Actual total mass fibers }}{\begin{array}{c}
\text { Calculated mass solids in volume } \\
\text { of solution added to cartridge }
\end{array}}
$$

$$
\text { Productivity }=\frac{\text { Actual total mass fibers }}{\text { Total area fibers } \times \text { Spinning time }}
$$

$\begin{aligned} & \% \text { relative } \\ & \text { crystallinity }\end{aligned}=100 \times \frac{\text { Area under curve }_{\text {sample peak }} \times \text { Mass }_{\text {sample }}}{\text { Area under curve }}$

Fabric thickness was measured using digital calipers and by averaging three measurements from different locations at the center of the fabric. Fluorescent fabrics were imaged using a Leica TCS NT/SP DMIRBE inverted confocal microscope (Leica Microsystems) located in the UW Keck Imaging Facility.

\section{Drug loading and release}

Drug loading was analyzed by dissolving approximately $5 \mathrm{mg}$ of fabrics in $20 \mathrm{~mL}$ of a mixture of 1:1 isopropanol:water in a glass vial. In vitro release was analyzed at sink conditions 
$(2.6 \mathrm{mg} / \mathrm{mL})$ in a 1:1 solution of isopropanol:water due to the limited solubility of LNG in water. ${ }^{10}$ Samples were incubated at $37^{\circ} \mathrm{C}$ in a rotational shaker and replenished with fresh media upon sampling. Triplicate samples were quantified using a Shimadzu Prominence LC20AD UV-HPLC system (Shimadzu Corporation, Kyoto, Japan). A Phenomenex Luna C18 column ( $5 \mu \mathrm{m}, 250.0 \times 4.6 \mathrm{~mm}$; Torrance, CA, USA) and LC Solutions software were used to analyze samples. A method for the dual analysis of TFV and LNG was developed using a gradient method with a mobile phase $\mathrm{A}(0.1 \%$ formic acid in water) and mobile phase $B$ (acetonitrile). The run consisted of 5 minutes $72 \% \mathrm{~A}, 5$ minutes ramp to $15 \% \mathrm{~A}$, 10 minutes $15 \% \mathrm{~A}, 5$ minutes ramp to $72 \% \mathrm{~A}$, and $5 \mathrm{~min}-$ utes $72 \% \mathrm{~A}$. The total time for the method was 30 minutes, with an oven temperature of $30^{\circ} \mathrm{C}$, flow rate of $1 \mathrm{~mL} / \mathrm{min}$, and injection volume of $20 \mu \mathrm{L}$. LNG and TFV were detected at $238 \mathrm{~nm}$ and $259 \mathrm{~nm}$, respectively, and at retention times of 18.5 minutes and 2.3 minutes, respectively. LNG had a linear standard range from $0.05 \mu \mathrm{g} / \mathrm{mL}$ to $100 \mu \mathrm{g} / \mathrm{mL}$ and $\mathrm{TFV}$ had a linear standard range from $0.01 \mu \mathrm{g} / \mathrm{mL}$ to $200 \mu \mathrm{g} / \mathrm{mL}$. The high-performance liquid chromatography (HPLC) method was also validated for $>95 \%$ recovery of a known concentration of both TFV and LNG, consistent retention time between standards and samples, and $>95 \%$ recovery in a spiked sample containing dissolved fibers and a known concentration of TFV and LNG.

\section{Cytotoxicity and HIV inhibition assays}

TZM-bl cells and HIV-1 BaL isolate were obtained from the National Institutes of Health (NIH) AIDS Research and Reference Reagent Program, NIAID (Germantown, MD, USA). TZM-bl cells are a HeLa-derived cell line that express CD4, CCR5, and CXCR4. ${ }^{19-22}$ Cells were plated in a black 96-well plate at a density of 5,000 cells/well (Corning, Corning, NY, USA) and maintained with Gibco ${ }^{\circledR}$ Dulbecco's Modified Eagle Medium (DMEM; Thermo Fisher Scientific) with 10\% fetal bovine serum (HyClone; Thermo Fisher Scientific), 1\% 100X penicillin/streptomycin (Thermo Fisher Scientific), and 1\% $200 \mathrm{mM}$ L-glutamine. Cells were incubated in $5 \% \mathrm{CO}_{2}$ at $37^{\circ} \mathrm{C}$ for 24 hours prior to exposure to drugs. Fabrics were sterilized by ultraviolet irradiation for 1 hour per side prior to use. Treatments were added at a volume of $50 \mu \mathrm{L}$. Cytotoxicity was analyzed by CellTiterBlue ${ }^{\circledR}$ Cell Viability Assay (Promega Corporation, Fitchburg, WI, USA) to assess TZM-bl cell viability after 48 hours of exposure to drug eluates from fabric release in cDMEM (24-hour release, in triplicate). Percent viability was calculated by normalizing to the average of media control wells $(n=9)$. For composite fabrics, the drug concentration shown applies to both the amount of $\mathrm{LNG}$ and of TFV (1:1 ratio). Positive control (12.5\% dimethyl sulfoxide) resulted in $4.6 \%$ viability (not shown).

For the HIV-infectious inhibition assay, $100 \mu \mathrm{L}$ of HIV-1 BaL (240 median tissue culture infective dose/well) was added to wells 1 hour after drug treatment (24-hour release eluates). Media was removed from wells after 48 hours and $100 \mu \mathrm{L}$ of phosphate-buffered saline (Thermo Fisher Scientific) and $100 \mu \mathrm{L}$ of Bright-Glo ${ }^{\text {TM }}$ Luciferase reagent (Promega Corporation) were added to wells. Inhibition of infectious activity was quantified by measuring luminescence on a plate reader (Tecan Group Ltd, Männedorf, Switzerland). Half maximal inhibitory concentration $\left(\mathrm{IC}_{50}\right)$ values of drug compounds were estimated using sigmoidal regression in GraphPad Prism (v 5.0; GraphPad Software, Inc, La Jolla, CA, USA).

\section{Statistical analysis}

Drug release is reported as mean \pm standard deviation, and values at each time point were compared using a two-way analysis of variance to compare all microarchitectures and a Bonferroni post-test to directly compare values for two individual microarchitectures. Two-sided tests were used at a significance level of $\alpha=0.05$ for all hypothesis testing. Statistical analyses were done in GraphPad Prism (v 5.0).

\section{Results}

\section{Properties of electrospun fabrics}

Free-surface electrospinning was used to prepare fabrics of different microarchitectures for the combined delivery of TFV and LNG (Figure 2). We observed that the addition of LNG and TFV, either alone or in combination, to the PVA polymer solution led to minimal differences in the properties of the solution (Table 1). The fabrics were macroscopically indistinguishable from each other, irrespective of the drugs loaded or composite microarchitecture (stacked, interwoven, or combined). Fabrics appeared white, were silky to the touch, flexible, and easily folded. SEM revealed fibers of $\sim 250 \mathrm{~nm}$ diameter with a smooth and rounded morphology (Table 2). All fabrics were also free of physical defects.

We achieved high encapsulation efficiencies of $>80 \%$ for all fabric formulations except for interwoven fabrics of TFV and LNG, where an artifact of the processing led to measured encapsulation efficiencies of $<50 \%$ and $>150 \%$ for TFV and LNG, respectively (Table 2). In this case, an extra valve and length of tubing present in the dual cartridge used to fabricate interwoven fabrics resulted in impeded flow of TFV solution onto the wire electrode (Figure 1B). Therefore, this particular cartridge design limited the deposition of 

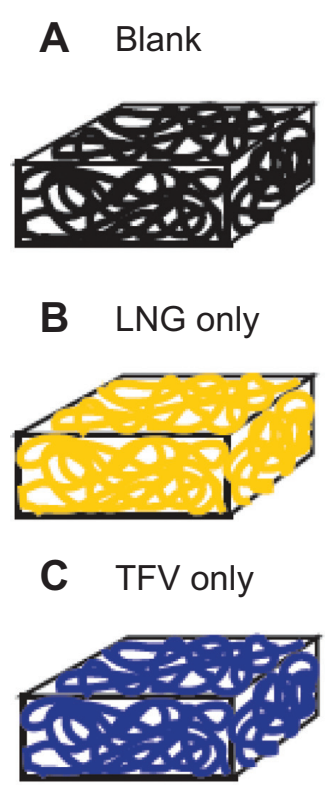
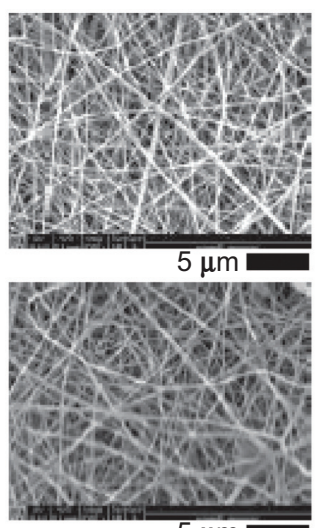

$5 \mu \mathrm{m}$

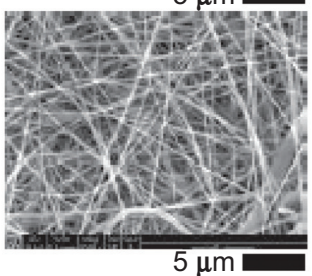

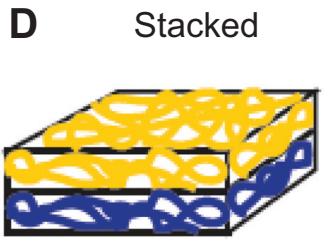
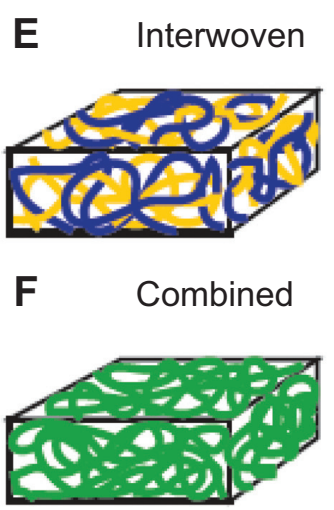

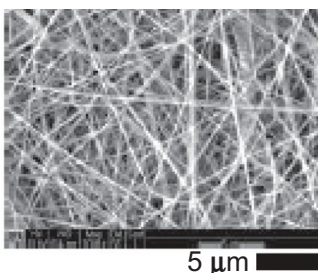

$5 \mu \mathrm{m}$

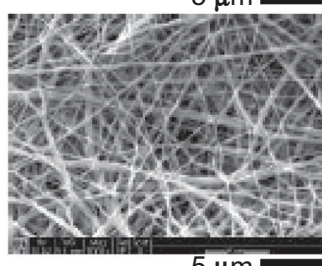

$5 \mu \mathrm{m}$

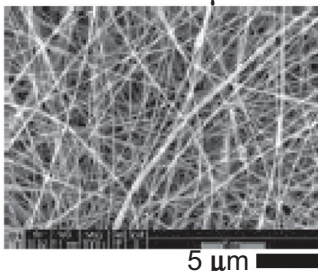

Figure 2 Electrospun fabric microarchitectures for topical delivery of single and combination drugs. Levonorgestrel (LNG) and tenofovir (TFV) were delivered alone (B, C) or combined using different composite microarchitectures (D-F). Vehicle control fabrics (blank) were prepared with only the PVA polymer (A). The figure shows the microscale rendering of idealized dispersions of LNG and TFV in fibers (left) and actual scanning electron micrographs (right) of representative fabrics that were produced.

TFV polymer solution on the were electrode and ultimately incorporated into the finished fibers. The differences in polymer solution deposition could not be accounted for in the predicted drug loading since the values assume equal flow, and is hence manifested as an artifact in the calculated percent encapsulation efficiencies.

\section{Differential scanning calorimetry analysis of electrospun fibers}

We used differential scanning calorimetry to characterize the solid dispersion of LNG and TFV in the final fabric products. The $\mathrm{T}_{\mathrm{m}}$ measured for PVA, $\mathrm{LNG}$, and TFV in all of our formulations was $195^{\circ} \mathrm{C}, 234^{\circ} \mathrm{C}$, and $270^{\circ} \mathrm{C}$, respectively (Figure 3 ). These values are consistent with values reported elsewhere. ${ }^{23-25}$

PVA is a semi-crystalline polymer and our PVA fabrics had a measured $\mathrm{T}_{\mathrm{g}}$ of $\sim 95^{\circ} \mathrm{C}$. Incorporation of LNG did not significantly change the $\mathrm{T}_{\mathrm{g}}$ of PVA fabrics. However, incorporation of TFV increased the $\mathrm{T}_{\mathrm{g}}$ of PVA fabrics by $>15^{\circ} \mathrm{C}$ to $\sim 110^{\circ} \mathrm{C}$, indicating an interaction between the TFV and PVA polymer. Crystalline TFV was detected in TFV-only, stacked and combined fabrics where the TFV loading was 7-14 wt\%, (Table 2). Interwoven fabrics had a lower TFV loading of $\sim 4 \mathrm{wt} \%$ and these fabrics did not have detectable TFV crystallinity (Table 2 ). TFV crystallinity in these fibers was expected since we made no attempt to neutralize the $\mathrm{pH}$ of our electrospinning solutions, which had an acidic $\mathrm{pH}$ where TFV precipitates. LNG-only fabrics with high drug loading of $17 \mathrm{wt} \%$ had detectable amounts of crystalline LNG of $\sim 1 \%$. We did not detect LNG crystallinity in the other fabrics, which also showed lower LNG loading. In general, electrospun drug loaded fabrics were prepared with overall minimal TFV and LNG crystallinity.

\section{Mechanical testing of electrospun fabrics}

All fabrics were tested to assess whether the incorporation of drugs into the PVA fibers or composite microarchitecture altered the mechanical properties of the resulting fabric.

Table I Composition and solution properties of fiber formulations

\begin{tabular}{|c|c|c|c|c|c|}
\hline Fabrics & $\begin{array}{l}\text { Density } \\
(\mathrm{g} / \mathrm{mL})\end{array}$ & $\begin{array}{l}\text { Conductivity } \\
(\mu \mathrm{S} / \mathrm{cm})\end{array}$ & pH & $\begin{array}{l}\text { Viscosity } \\
\text { Pa.s, g }=10 \mathrm{~s}^{-1}\end{array}$ & $\begin{array}{l}\text { Surface } \\
\text { tension }(\mathrm{mN} / \mathrm{m})\end{array}$ \\
\hline PVA only & 1.10 & 573 & 5.58 & 0.45 & 59.0 \\
\hline LNG only (20 wt\%) & 1.12 & 649 & 5.58 & 0.46 & 58.0 \\
\hline TFV only (20 wt\%) & $\mathrm{I} .08$ & 527 & 3.85 & 0.53 & 59.4 \\
\hline Combined TFV/LNG (20 wt\% each) & 1.05 & 438 & 4.01 & 0.45 & 55.3 \\
\hline
\end{tabular}

Abbreviations: LNG, levonorgestrel; PVA, polyvinyl alcohol; TFV, tenofovir. 
Table 2 Properties of drug-loaded electrospun fibers

\begin{tabular}{|c|c|c|c|c|c|c|c|}
\hline \multirow[t]{2}{*}{ Fabrics } & \multicolumn{2}{|c|}{ EE (\%), loading (wt\%) } & \multirow[t]{2}{*}{$\begin{array}{l}\text { Fiber diameter } \\
(\mathrm{nm})\end{array}$} & \multirow[t]{2}{*}{$\begin{array}{l}\text { Fiber yield } \\
\text { (\%) }\end{array}$} & \multirow[t]{2}{*}{$\begin{array}{l}\text { Productivity } \\
\left(\mathrm{g} / \mathrm{m}^{2} / \mathrm{hr}\right)\end{array}$} & \multicolumn{2}{|c|}{$\begin{array}{l}\text { Drug } \\
\text { crystallinity (\%) }\end{array}$} \\
\hline & LNG & TFV & & & & LNG & TFV \\
\hline PVA only & - & - & $208 \pm 106$ & 39.4 & 12.9 & - & - \\
\hline \multicolumn{8}{|c|}{ Single drug fabrics } \\
\hline LNG only & $100 \pm 1.1(16.7 \%)$ & - & $260 \pm 105$ & 33.8 & 18.0 & $1.2 \%$ & - \\
\hline TFV only & - & $85 \pm 1.3(14.3 \%)$ & $267 \pm 176$ & 49.9 & 18.0 & - & $0.2 \%$ \\
\hline \multicolumn{8}{|c|}{ Multidrug composite fabrics } \\
\hline Stacked & $114 \pm 9.4(9.6 \%)$ & $83 \pm 1.1(6.9 \%)$ & $25 I \pm 107$ & 67.9 & 26.6 & nd & $0.2 \%$ \\
\hline Interwoven & $166 \pm 9.6(13.9 \%)$ & $44 \pm 2.4(3.7 \%)$ & $303 \pm 123$ & 31.9 & 16.8 & nd & nd \\
\hline Combined & $82 \pm 1.5(11.6 \%)$ & $93 \pm 1.9(14.3 \%)$ & $25 I \pm 83$ & 39.7 & 18.7 & nd & $2.3 \%$ \\
\hline
\end{tabular}

Abbreviations: LNG, levonorgestrel; PVA, polyvinyl alcohol; TFV, tenofovir; EE, encapsulation efficiency.

TFV incorporation into fibers (Figure 4) did not affect the Young's modulus or tensile strength compared with blank PVA fibers. However, incorporating LNG lowered the Young's modulus and tensile strength by approximately threefold (Figure 4). As expected, the three composite microarchitectures had lower Young's moduli and tensile strengths than PVA alone, due to the presence of both LNG and TFV in these samples. Young's modulus and tensile strength does not appear to vary based on the underlying microarchitecture.

\section{Visualization of composite microarchitectures}

Confocal microscopy was used to visualize the stacked, interwoven, and combined composite microarchitectures by

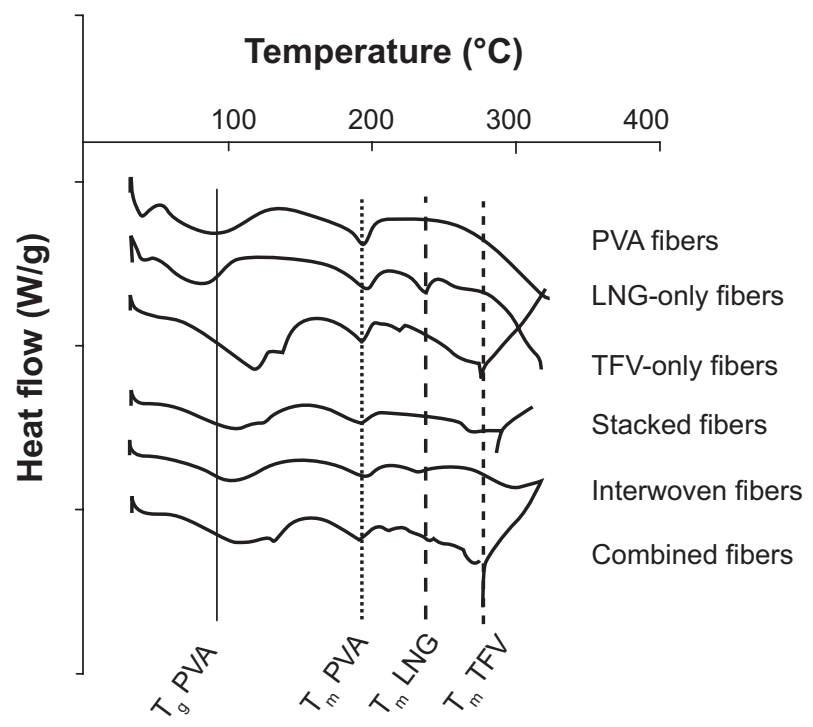

Figure 3 Thermograms of electrospun fibers of varying microarchitectures, as determined by differential scanning calorimetry.

Note: The $T_{g}$ and $T_{m}$ peaks of the pure components are indicated with vertical lines.

Abbreviations: LNG, levonorgestrel; PVA, polyvinyl alcohol; TFV, tenofovir. co-loading the fibers with drugs and different fluorophores. Alexa Fluor ${ }^{\circledR} 488$ was co-loaded with LNG, whereas Alexa Fluor ${ }^{\circledR} 555$ was co-loaded with TFV. LNG-only fibers were observed only in the green channel (Figure 5A), TFV-only fibers were observed only in the red channel (Figure 5B), and co-localization of the fluorophores (and drugs) was visualized as an orange color in the merged channel. The stacked composites were imaged at the interface of the LNG/TFV layers, where we observed distinct red and green fibers and no fluorescence co-localization (Figure 5C). In contrast to the stacked composites, the interwoven fabrics showed intermingling LNG-only and TFV-only fibers, as well as orange fibers in the merged fluorescent images (Figure 5D). This assortment of fiber compositions may be due to the ability of solutions to mix at the orifice before fiber formation. The interwoven fabrics showed fewer red fibers compared with the number of green fibers, which supports our earlier observation of low TFV loading in this fabric composition (Table 2). Visualization of orange fluorescence in the combined microarchitectures confirmed the co-localization of

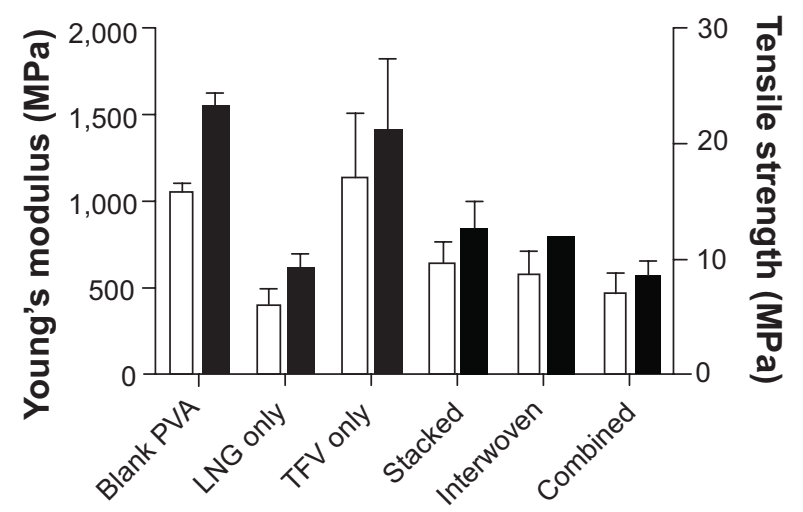

Figure 4 Mechanical properties, including Young's modulus and tensile strength, for electrospun fabrics. White bars represent Young's modulus data and black bars represent tensile strength. Values are reported as mean \pm standard deviation for $\mathrm{n}=5$ samples.

Abbreviations: LNG, levonorgestrel; PVA, polyvinyl alcohol; TFV, tenofovir. 
Alexa Fluor $^{\circledR} 488$

(LNG)

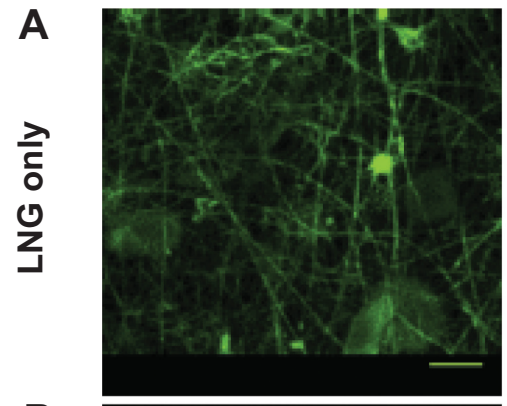

B

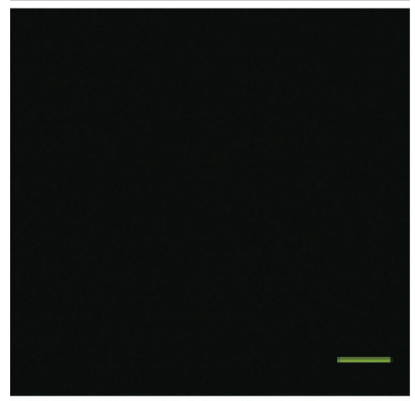

C

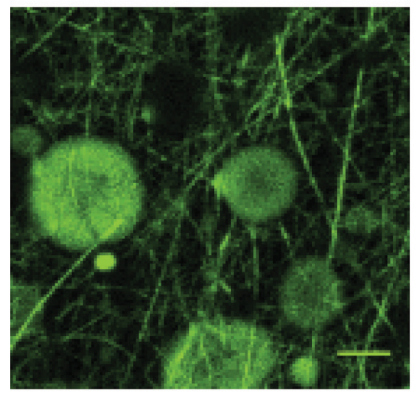

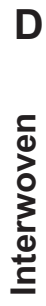

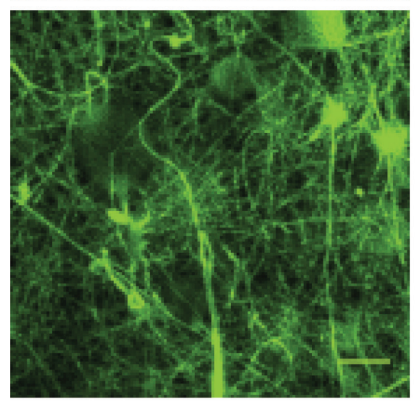

E

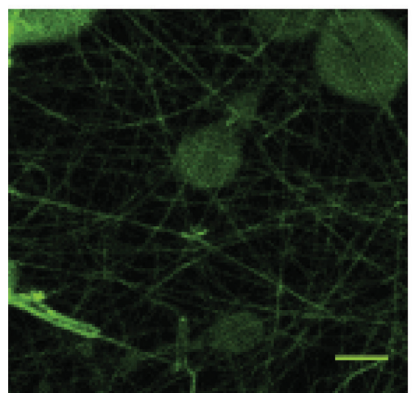

Alexa Fluor ${ }^{\circledR} 555$

(TFV)
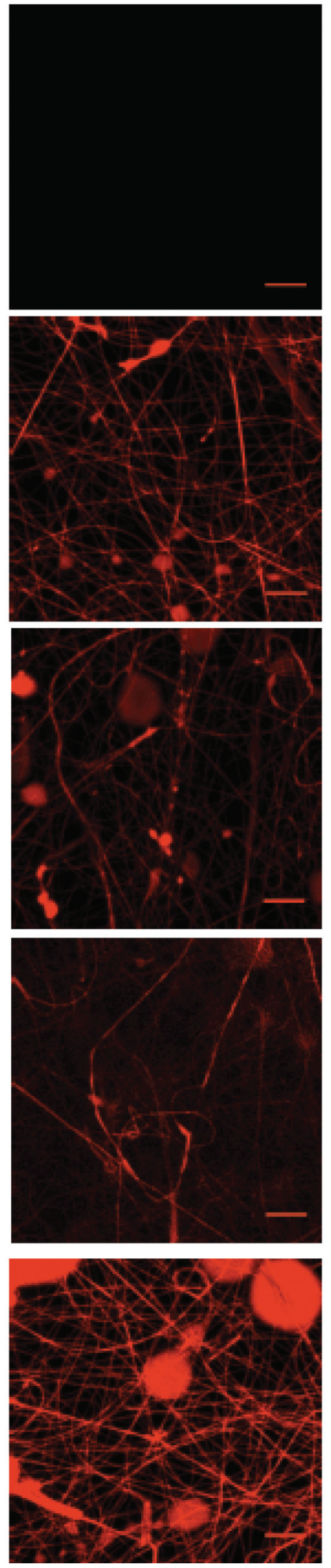

488/555 merge

(LNG + TFV)
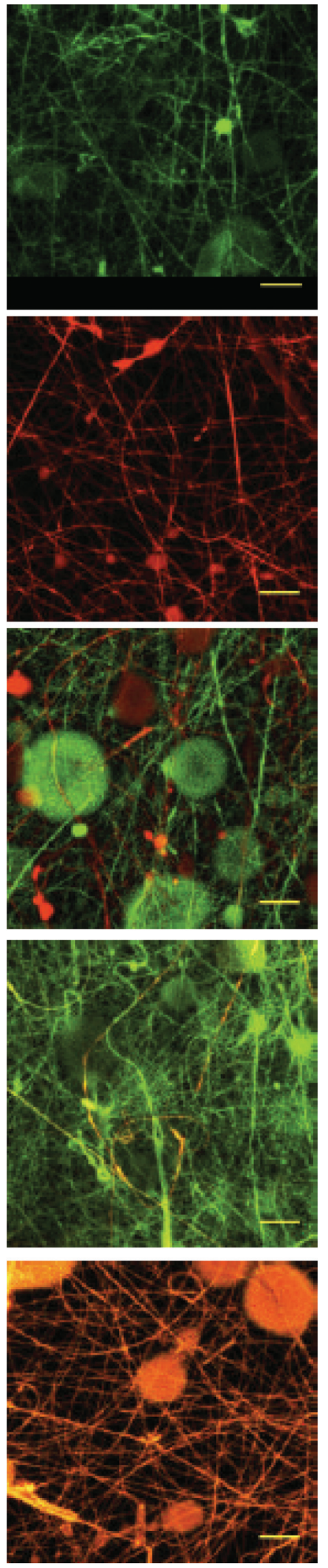

Figure 5 Confocal images of fabric microarchitectures. Levonorgestrel (LNG) and tenofovir (TFV) were fabricated alone (A and B, respectively) or combined in different composites (C-E). To visualize the underlying fabric microarchitectures, LNG solutions were co-loaded with Alexa Fluor ${ }^{\circledast 88}$ (Thermo Fisher Scientific, Waltham, MA, USA) and TFV solutions were co-loaded with Alexa Fluor 555 (Thermo Fisher Scientific). Fluorescently labeled fibers were analyzed with a confocal laser scanning microscope (Leica TCS NT/SP, Leica Microsystems GmbH, Wetzlar, Germany). Samples were scanned for Alexa Fluor ${ }^{\circledR} 488$ (green channel, left) and Alexa Fluor ${ }^{\circledR}$ 555 (red channel, center), then the two channels were merged (right) using Image $\left.\right|^{17}$ software to detect fluorescence co-localization. Equal exposure time was used for all images. Scale bar $=10 \mu \mathrm{m}$. 
both fluorophores (Figure 5E). For the fluorescent microscopy, fabrics were collected onto a glass slide that altered the electric field and caused polymer droplets to collect, which was observed in many of our images.

Cross-sectional SEM imaging did not reveal obvious differences in the composite microarchitecture in terms of distinct layering based on fiber size or morphology alone. Images of control fabrics (Figure 6A-C) show a single layer of fibers with similar size and morphology. Though the stacked composite (Figure 6D) is comprised of distinct layers of TFV- and LNG-loaded fibers, this microarchitecture is not visible by SEM, as the TFV- and LNG-only fibers have very similar morphology. Further, no distinct layering or varying fiber morphologies was visible in the cross-section of the interwoven or combined composites (Figure 6E and F).

\section{In vitro release of TFV and LNG}

We assessed the role of composite microarchitecture, drug loading, and fabric thickness on the release of TFV and LNG in vitro. Despite the similar actual drug loading of TFV and LNG in the PVA fibers, LNG displayed much slower release kinetics. TFV reached $100 \%$ release within 30 minutes whereas LNG did not reach $100 \%$ release until 4 hours (Figure 7A and B). The significant difference in release is attributed to the highly hydrophobic nature of fabrics containing LNG, which appeared to take hours as opposed to minutes to wet and dissolve in the release media compared with TFV-only fabrics (data not shown).

We next compared the co-release of TFV and LNG from the three distinct composite microarchitectures to the release kinetics of TFV- or LNG-only fabrics. The release of TFV was similar in the control, stacked, and interwoven microarchitectures, where we observed $\sim 50 \%$ TFV release after 5 minutes and $100 \%$ release after 30 minutes. In contrast, release of TFV from the combined microarchitecture was much slower, with $\sim 50 \% \mathrm{TFV}$ release after 30 minutes and 100\% release after 60 minutes (Figure 7B and D). Percentage TFV release measured at 5, 15, and 30 minutes was significantly $(P<0.05)$ less from the combined microarchitecture than from the stacked and interwoven microarchitectures. LNG release was similar for all microarchitectures, and showed complete release within 4 hours (Figure 7A and C). We observed that TFV release was two-fold slower from the combined fabrics than from the TFV-only fabrics, despite similar TFV loading (14.3 wt\%) (Figure 7B). In contrast, percent TFV release from the interwoven fabrics was similar to TFV-only fabrics, despite the high LNG loading. Therefore, the combination of both drugs in a single fiber (combined microarchitecture), but not the stacked or interwoven microarchitectures, had an impact on TFV release.

We also prepared PVA fabrics containing relative loadings of TFV to LNG of 2000 to 1, a ratio that is better representative of the potential dosing requirements of these two drugs. ${ }^{26,27}$ This new ratio also allowed us to test the hypothesis
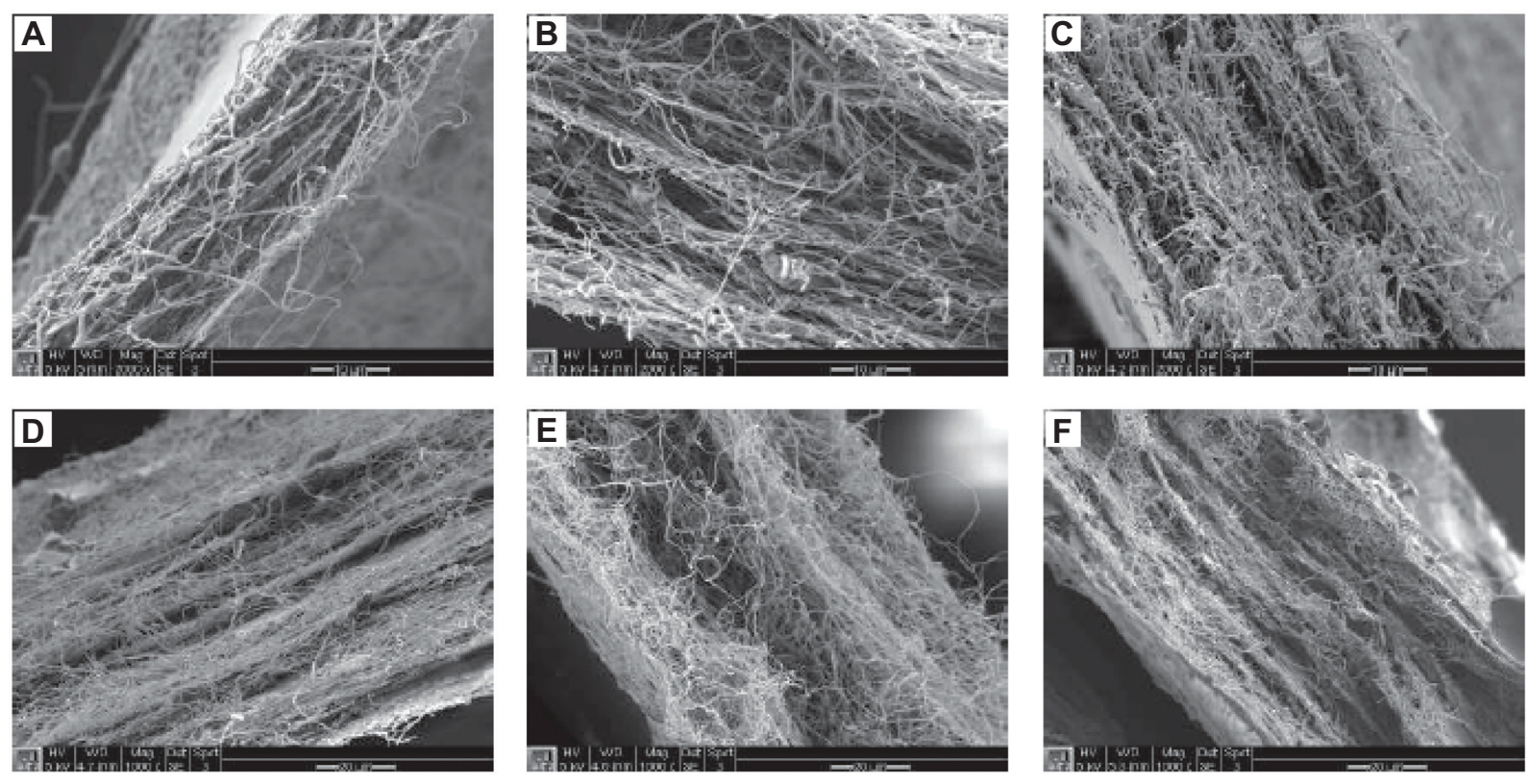

Figure 6 Cross-sectional scanning electron micrographs of electrospun fabrics. Samples were cryo-fractured using liquid nitrogen to achieve a clean break, and then imaged to observe microarchitectural differences in the z-plane. (A) PVA only, (B) LNG-only, (C) TFV-only, (D) stacked, (E) interwoven, and (F) combined images of composite fabrics.

Notes: Images A-C have a scale bar of $10 \mu \mathrm{m}$. Images D-F have a scale bar of $20 \mu \mathrm{m}$. 
A

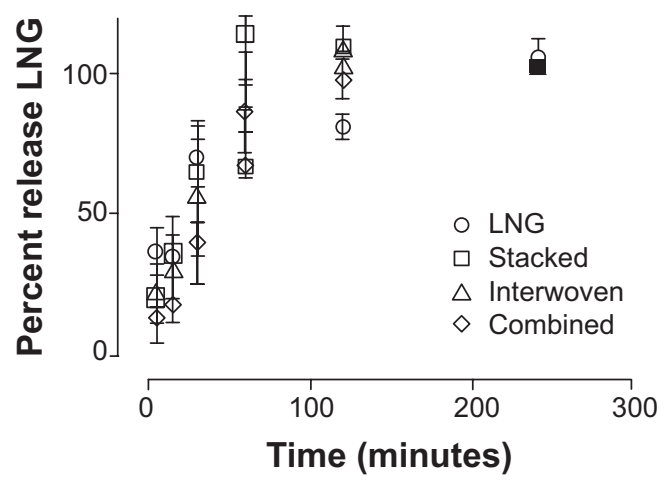

C

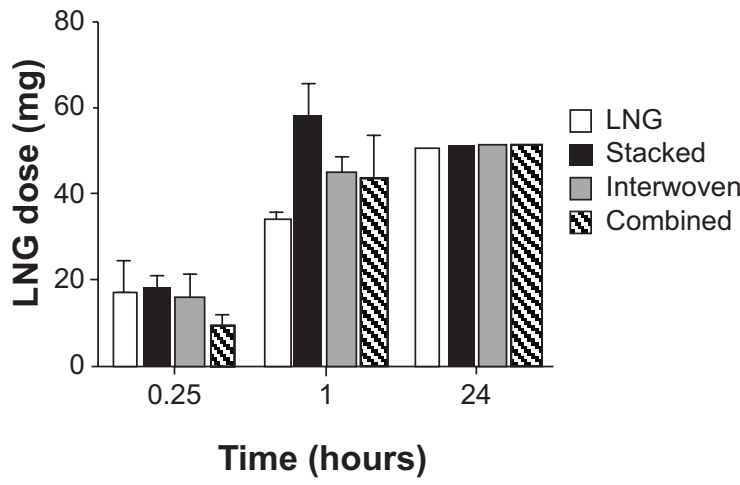

E

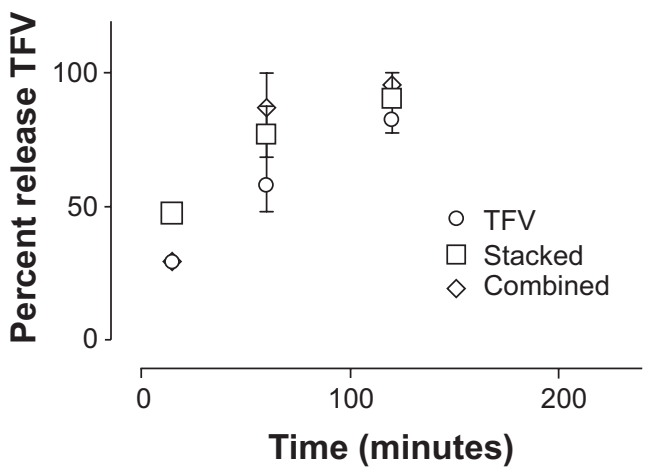

B

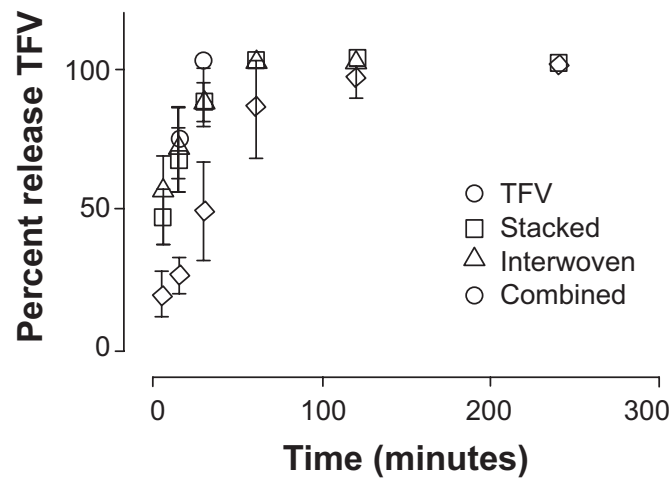

D

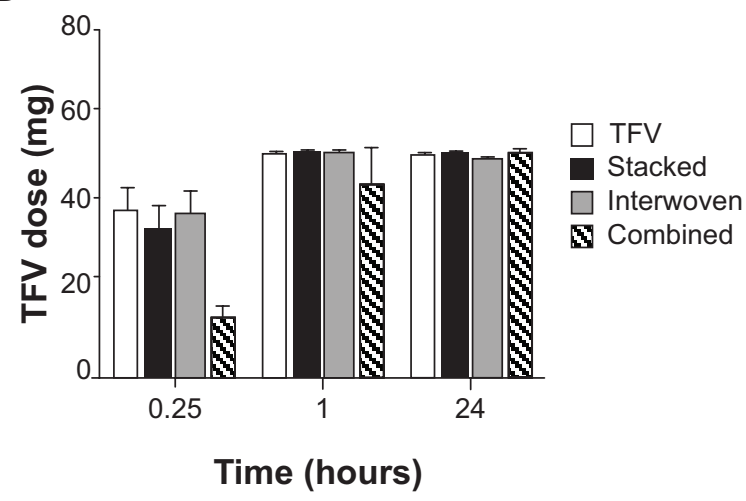

$\mathbf{F}$

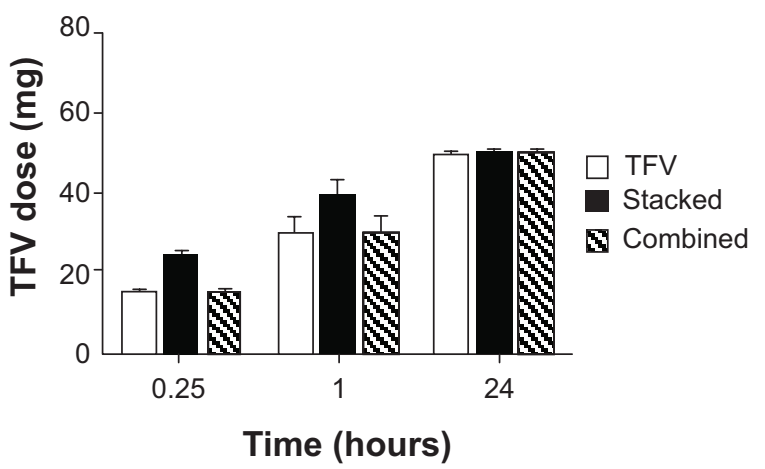

Figure $\mathbf{7}$ In vitro release profiles (A, B, E) and dosage profiles (C, D, F) of levonorgestrel (LNG) and tenofovir (TFV) delivered from different composite fibers. Fabrics were prepared at I:I equal TFV:LNG mass loading (A-D) or at 2000:I TFV:LNG mass loading (E, F). The delivered dose was calculated at each time point using the percent release at that time and an assumed delivery of $500 \mathrm{mg}$ of total fabric.

Note: Values are reported as mean and standard deviation for $n=3$.

that decreasing the LNG loading would reduce the hindered release of TFV. Indeed, the combined microarchitecture with this ratio had similar a TFV release to the TFV-only or stacked microarchitecture, with all three compositions achieving $100 \%$ release after 2 hours (Figure 7E and F). We did not measure LNG release from these fabrics because the low LNG loading was below the detection limit of our analytical method.

As previously reported by Jannesari et al increasing the thickness of electrospun fabrics can slow the release of a single agent. ${ }^{28}$ Using the stacked microarchitecture, we prepared three different thicknesses ranging from $110 \mu \mathrm{m}$ to $180 \mu \mathrm{m}$ (Figure $8 \mathrm{~A})$. TFV release was slowest from the thickest fabric (Figure 8B), which was probably due to the increased wetting time for the thicker fabrics. In contrast, we did not observe a difference in LNG release with changes in mat thickness (Figure 8C).

\section{In vitro cytotoxicity and inhibition of HIV-I BaL infection}

The activity and toxicity of the PVA, LNG-only, TFV-only, stacked, and combined fabrics were evaluated using in vitro assays for cytotoxicity and HIV infection. PVA and 
A
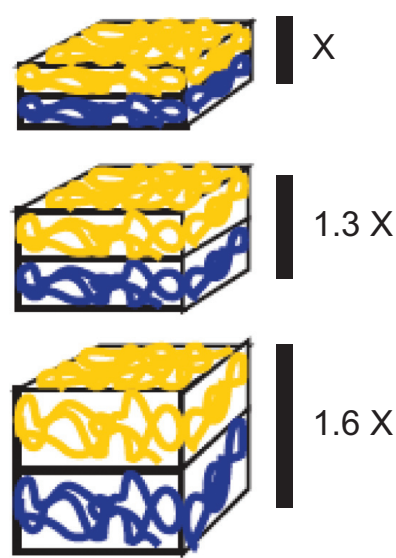

B

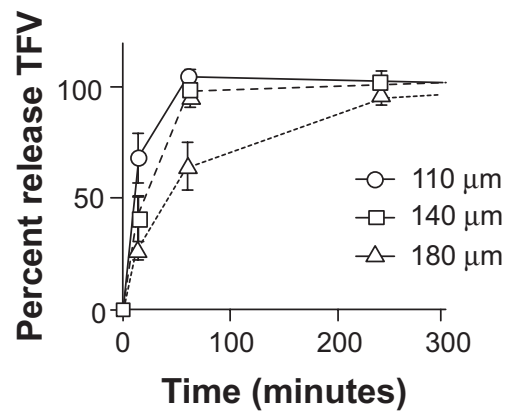

C

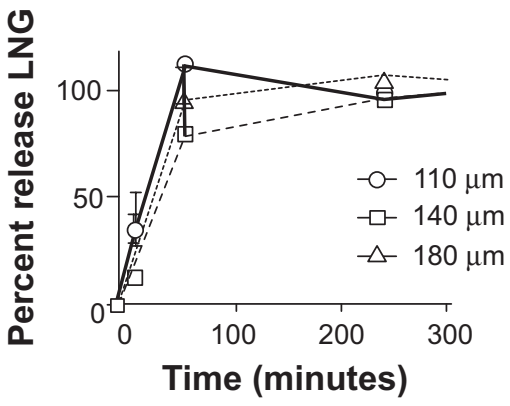

Figure 8 Effect of levonorgestrel (LNG) and tenofovir (TFV) release from stacked composites of different thickness. (A) Schematic of stacked composite fibers with increasing thicknesses, and in vitro release profiles of (B) TFV and (C) LNG from stacked composite fibers of varying thickness.

TFV-only fabrics were found to be nontoxic to TZM-bl cells at all concentrations tested (Figure 9A). Fabrics containing LNG were toxic at concentrations of $5 \mu \mathrm{M} \mathrm{LNG}$ and higher (Figure 9A). Based on these results and the more clinically relevant dosing, antiviral HIV activity was assessed using fabrics with the lower loading of LNG $(0.0067 \mathrm{wt} \%)$. The TFV-only, stacked, and combined fabrics had $\mathrm{IC}_{50}$ values of 2.2, 1.7, and 2.9 $\mu \mathrm{M}$ TFV, respectively (Figure 9B). There was no statistically significant difference in antiviral activity between TFV in the fabrics of varying microarchitectures, and the free drug, which has a measured $\mathrm{IC}_{50}$ of $1.8 \mu \mathrm{M}$ and is similar to previously reported values. ${ }^{14}$ Therefore, the activity of TFV was preserved through the electrospinning process and was unaffected by composite geometry.

\section{Discussion}

Here, we present the first report of combination drug-eluting fabrics with high drug loading and varying microarchitectures that have been fabricated using a free-surface, productionscale electrospinning instrument. Electrospinning allows for the formation of nanofibers from a drug-loaded polymer solution by subjecting the solution to an applied voltage, which charges the fluid and causes the eruption of a jet. The charged fluid jet is accelerated in an electric field toward the region of lower potential, and polymer entanglement prevents the break up of the stream while the solvent evaporates and results in fiber formation. While the differences in the release properties varied with the microarchitecture, the macroscopic appearance and mechanical properties were unaltered. The ability to realize different microarchitectures without
A

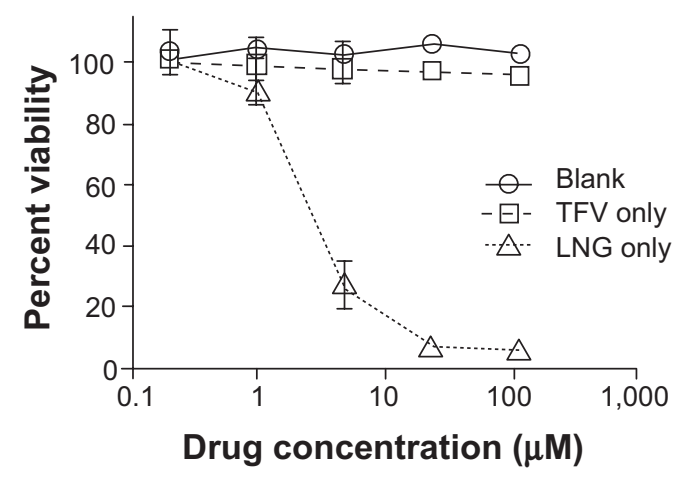

B

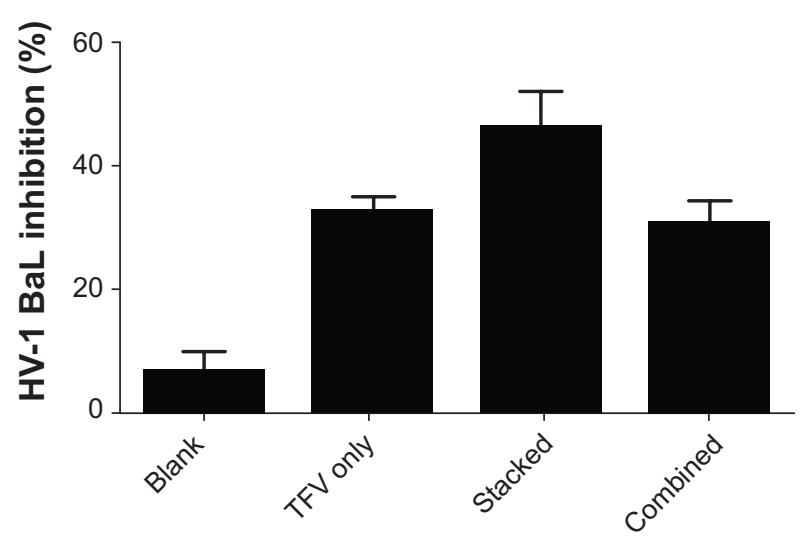

Figure 9 Cytotoxicity and HIV antiviral activity of drug-loaded fabrics of varying microarchitecture. (A) Cytotoxicity of blank, tenofovir (TFV)-only, and levonorgestrel (LNG)-only fabrics in TZM-bl cells. (B) Inhibition of HIV-I BaL infection of TZM-bl cells by blank, TFV-only, stacked, and combined fibers at a delivered TFV concentration of I $\mu$ M (the half maximal inhibitory concentration of free TFV was I.8 $\mu \mathrm{M}$ ). Due to toxicity observed for LNG, fabrics with a TFV:LNG ratio of 2000:I were used for testing antiviral activity. Blank fibers were tested based on equivalent polymer concentrations used for TFV-only fabrics.

Note: Values are reported as mean \pm standard deviation for $n=3$. 
changing the visual or tactile properties of the materials may support better designs of topical delivery systems for both biological efficacy and user acceptability. ${ }^{29}$ The ability to vary the microstructure on a production-scale instrument may also prove to be useful in a number of drug-delivery applications, including wound healing and tissue engineering. ${ }^{30-32}$

We observed differences in the release of TFV from fabrics of varied loading and microarchitecture (Figure 7B and E) but not in the release of the more hydrophobic LNG (Figure 7A). The slower release of TFV seen with increasing fabric thickness is likely due to slower wetting and increased distance for drug diffusion through the fiber matrix. The release of LNG was largely unchanged by the microarchitecture and fabric thickness, where the hydrophobicity and dissolution of the fabric may be the limiting factor in release. We suspect that the slowed release of TFV in the combined fabrics was due to the depletion of TFV in the interfacial layer between the dissolving front and the solvent, leading to the creation of a surface layer rich in LNG through which the TFV and PVA must dissolve. ${ }^{33}$ However, the versatile nature of electrospinning may allow for the decoupling of complex, multicomponent systems into single component systems via stacked or interwoven architectures that may be easier to control drug release.

The composite microarchitectures utilized in these experiments could be tailored for different types of vaginal topical delivery systems. In concept, the fabrics would be inserted vaginally and then release drugs into the local tissue at appropriate doses of TFV and LNG to prevent both HIV-1 acquisition and unintended pregnancy. The fibers would then dissolve and be eliminated, or be cleared by the flow of vaginal mucus. For example, the stacked composite could potentially be used to asynchronously deliver drugs in the vaginal mucosal environment by incorporating a layer that provides rapid drug release toward the mucosal tissue and a layer for sustained drug delivery toward the vaginal lumen. The combined microarchitecture has the advantage of manufacturing ease, since only one solution is prepared and electrospun, but may not allow for the decoupling of release profiles for co-delivered drugs. Pending improved drugloading efficiency, the interwoven microarchitecture may allow for the independent release profiles of physicochemically diverse drugs. The highly loaded fabrics developed in this study could potentially be used as a fast-acting pericoital product for the prevention of both HIV-1 transmission and unwanted pregnancy. However, extensive testing regarding safety, toxicity, effectiveness, and user perception needs to be performed to clearly establish the potential of these fabrics.

\section{Conclusion}

We prepared composite fabrics containing high loading of TFV and LNG in three distinct microarchitectures by freesurface electrospinning using a production-scale instrument. We observed that the release of LNG from composite fabrics is largely unaffected by geometry, thickness, and drug loading. However, TFV release was slower when combined in the same fiber with LNG as well as with increasing fabric thickness. The antiviral activity of TFV against HIV-BaL infection in TZM-bl cells was similar to that of the unformulated drug, and was not affected by the microarchitectures of the composite fabrics. Our findings are significant because they are the first, to inform the design and production of composite electrospun fabrics with high drug loading for the co-delivery of physicochemically diverse drugs that may be useful in topical delivery systems for multipurpose prevention.

\section{Acknowledgments}

The research was supported by funding from the Bill and Melinda Gates Foundation (OPP1067729) and a NIH/NIAID grant (AI098648) to KAW. AKB and EAK were supported by National Science Foundation Graduate Research Fellowships, and AKB was also supported by an NIH Molecular Medicine Training Fellowship (5T32GM095421-03). We thank Dr. Shih-Feng Chou for assistance with mechanical testing of electrospun fabrics.

\section{Disclosure}

The authors declare no conflicts of interest in this work.

\section{References}

1. Mayer LD, Janoff AS. Optimizing combination chemotherapy by controlling drug ratios. Mol Interv. 2007;7(4):216-223.

2. Sosnik A, Chiappetta DA. Drug delivery systems in HIV pharmacotherapy: what has been done and the challenges standing ahead. J Control Release. 2009;138(1):2-15.

3. Wu P, Grainger DW. Drug/device combinations for local drug therapies and infection prophylaxis. Biomaterials. 2006;27(11):2450-2467.

4. Greco F, Vicent MJ. Combination therapy: opportunities and challenges for polymer-drug conjugates as anticancer nanomedicines. Adv Drug Deliv Rev. 2009;61(13):1203-1213.

5. Sengupta S, Eavarone D, Capila I, et al. Temporal targeting of tumour cells and neovasculature with a nanoscale delivery system. Nature. 2005; 436(7050):568-572.

6. Zhang L, Radovic-Moreno AF, Alexis F, et al. Co-delivery of hydrophobic and hydrophilic drugs from nanoparticle-aptamer bioconjugates. Chem Med Chem. 2007;2(9):1268-1271.

7. Li X, Kanjwal MA, Lin L, Chronakis IS. Electrospun polyvinyl-alcohol nanofibers as oral fast-dissolving delivery system of caffeine and riboflavin. Colloids Surf B Biointerfaces. 2013;103:182-188.

8. Zhang YZ, Wang X, Feng Y, Li J, Lim CT, Ramakrishna S. Coaxial electrospinning of (fluorescein isothiocyanate-conjugated bovine serum albumin)-encapsulated poly(epsilon-caprolactone) nanofibers for sustained release. Biomacromolecules. 2006;7(4):1049-1057.

9. Prabaharan M, Jayakumar R, Nair S. Electrospun nanofibrous scaffoldscurrent status and prospects in drug delivery. Adv Polym Sci. 2012; 246:241-262. 
10. Friend DR, Doncel GF. Combining prevention of HIV-1, other sexually transmitted infections and unintended pregnancies: Development of dual-protection technologies. Antiviral Res. 2010;88 Suppl 1: S47-S54.

11. De Clercq E. Acyclic nucleoside phosphonates: past, present and future. Bridging chemistry to HIV, HBV, HCV, HPV, adeno-, herpes-, and poxvirus infections: the phosphonate bridge. Biochemical Pharmacol. 2007;73(7):911-922.

12. Chrisman CE, Curtis KM, Mohllajee AP, Gaffield ME, Peterson HB. Effective use of hormonal contraceptives: Part II: Combined hormonal injectables, progestogen-only injectables and contraceptive implants. Contraception. 2006;73(2):125-133.

13. Huang C, Soenen SJ, van Gulck E, et al. Electrospun cellulose acetate phthalate fibers for semen induced anti-HIV vaginal drug delivery. Biomaterials. 2012;33(3):962-969.

14. Ball C, Krogstad E, Chaowanachan T, Woodrow K. Drug-eluting fibers for HIV-1 inhibition and contraception. PloS One. 2012;7(11): e49792.

15. Okuda T, Tominaga K, Kidoaki S. Time-programmed dual release formulation by multilayered drug-loaded nanofiber meshes. J Control Release. 2010;143(2):258-264.

16. Xu X, Chen X, Wang Z, Jing X. Ultrafine PEG-PLA fibers loaded with both paclitaxel and doxorubicin hydrochloride and their in vitro cytotoxicity. Eur J Pharm Biopharm. 2009;72(1):18-25.

17. Rasband WS. ImageJ [software]. Bethesda, MD: US National Institutes of Health; 1997-2014. Available from: http://imagej.nih.gov/ij/. Accessed April 24, 2014.

18. Rawlinson CF, Williams AC, Timmins P, Grimsey I. Polymer-mediated disruption of drug crystallinity. Int J Pharm. 2007;336(1):42-48.

19. Takeuchi Y, McClure MO, Pizzato M. Identification of gammaretroviruses constitutively released from cell lines used for human immunodeficiency virus research. J Virol. 2008;82(24):12585-12588.

20. Derdeyn CA, Decker JM, Sfakianos JN, et al. Sensitivity of human immunodeficiency virus type 1 to the fusion inhibitor T-20 is modulated by coreceptor specificity defined by the V3 loop of gp120. J Virol. 2000;74(18):8358-8367.

21. Platt EJ, Wehrly K, Kuhmann SE, Chesebro B, Kabat D. Effects of CCR5 and CD4 cell surface concentrations on infections by macrophagetropic isolates of human immunodeficiency virus type 1 . J Virol. 1998;72(4):2855-2864.
22. Wei X, Decker JM, Liu H, et al. Emergence of resistant human immunodeficiency virus type 1 in patients receiving fusion inhibitor (T-20) monotherapy. Antimicrob Agents Chemother. 2002;46(6):1896-1905.

23. Shao C, Kim KY, Gong J, Ding B, Lee DR, Park SJ. Fiber mats of poly(vinyl alcohol)/silica composite via electrospinning. Mater Lett. 2003;57(9-10):1579-1584.

24. Dasaratha Dhanaraju M, Vema K, Jayakumar R, Vamsadhara C. Preparation and characterization of injectable microspheres of contraceptive hormones. Int J Pharm. 2003;268(1-2):23-29.

25. Johnson TJ, Gupta KM, Fabian J, Albright TH, Kiser PF. Segmented polyurethane intravaginal rings for the sustained combined delivery of antiretroviral agents dapivirine and tenofovir. Eur J Pharm Sci. 2010; 39(4):203-212

26. Gallo MF, Nanda K, Grimes DA, Schulz KF. Twenty micrograms vs $>20$ microg estrogen oral contraceptives for contraception: systematic review of randomized controlled trials. Contraception. 2005;71(3):162-169.

27. Nicol MR, Kashuba AD. Pharmacologic opportunities for HIV prevention. Clin Pharmacol Ther. 2010;88(5):598-609.

28. Jannesari M, Varshosaz J, Morshed M, Zamani M. Composite poly(vinyl alcohol)/poly(vinyl acetate) electrospun nanofibrous mats as a novel wound dressing matrix for controlled release of drugs. Int J Nanomedicine. 2011;6:993-1003.

29. Morrow K, Rosen R, Richter L, et al. The acceptability of an investigational vaginal microbicide, PRO $2000 \mathrm{Gel}$, among women in a phase I clinical trial. J Womens Health (Larchmt). 2003;12(7):655-666.

30. Miao YE, Zhu H, Chen D, Wang R, Tjiu WW, Liu T. Electrospun fibers of layered double hydroxide/biopolymer nanocomposites as effective drug delivery systems. Mater Chem Phys. 2012;134(2-3):623-630.

31. Chen DW, Liao JY, Liu SJ, Chan EC. Novel biodegradable sandwichstructured nanofibrous drug-eluting membranes for repair of infected wounds: an in vitro and in vivo study. Int J Nanomedicine. 2012;7: 763-771.

32. Goh YF, Shakir I, Hussain R. Electrospun fibers for tissue engineering, drug delivery, and wound dressing. J Mater Sci. 2013;48(8): 3027-3054.

33. Craig DQ. The mechanisms of drug release from solid dispersions in water-soluble polymers. Int J Pharm. 2002;231(2):131-144.
International Journal of Nanomedicine

\section{Publish your work in this journal}

The International Journal of Nanomedicine is an international, peerreviewed journal focusing on the application of nanotechnology in diagnostics, therapeutics, and drug delivery systems throughout the biomedical field. This journal is indexed on PubMed Central, MedLine, CAS, SciSearch ${ }^{\circledR}$, Current Contents ${ }^{\circledR} /$ Clinical Medicine,

\section{Dovepress}

Journal Citation Reports/Science Edition, EMBase, Scopus and the Elsevier Bibliographic databases. The manuscript management system is completely online and includes a very quick and fair peer-review system, which is all easy to use. Visit http://www.dovepress.com/ testimonials.php to read real quotes from published authors. 